\title{
Intimate partner violence and mental health in Bolivia
}

Dominique Meekers ${ }^{1,2^{*}}$, Sarah C Pallin ${ }^{1}$ and Paul Hutchinson ${ }^{1}$

\begin{abstract}
Background: Latin America has among the highest rates of intimate partner violence. While there is increasing evidence that intimate partner violence is associated with mental health problems, there is little such research for developing countries. The purpose of this paper is to examine the relationship between Bolivian women's experiences with physical, psychological, and sexual intimate partner violence and mental health outcomes.

Methods: This study analyzes data from the 2008 Bolivia Demographic and Health Survey. 10,119 married or cohabiting women ages 15-49 are included in the analysis. Probit regression models are used to assess the association between intimate partner violence and mental health, after controlling for other demographic factors and partner characteristics. The questionnaire uses selected questions from the SRQ-20 to measure symptoms of mental health problems.
\end{abstract}

Results: Intimate partner violence is common in Bolivia, with $47 \%$ of women experiencing some type of spousal abuse in the 12 months before the survey. Women exposed to physical spousal violence in the past year are more likely to experience symptoms of depression, anxiety, psychogenic non-epileptic seizures, and psychotic disorders, after controlling for other demographic and partner characteristics. Women who experienced sexual abuse by a partner are most likely to suffer from all mental health issues. Psychological abuse is also associated with an increased risk of experiencing symptoms of depression, anxiety, and psychogenic seizures. Women who experienced only psychological abuse report mental health problems similar to those who were physically abused.

Conclusion: This study demonstrates an urgent need for research on the prevalence and health consequences of psychological abuse in developing countries. Our findings highlight the need for mental health services for victims of intimate partner violence. Because physical and psychological violence are often experienced concurrently, it is recommended that health providers who are treating victims of physical intimate partner violence also screen them for symptoms of potential mental health problems and refer them to appropriate mental health services.

Keywords: Domestic violence, Intimate partner violence, Psychological abuse, Mental health, Bolivia, Latin America

\section{Introduction}

There is increasing awareness that intimate partner violence (IPV) affects all societies. However, the prevalence of IPV, including physical, sexual, and psychological violence, varies considerably. Latin America, including Bolivia, has above-average rates of domestic violence [1-5]. A substantial body of research shows that domestic violence is associated with physical health problems $[1,2,5-9]$. While there is ample research on the potential

\footnotetext{
* Correspondence: dmeekers@tulane.edu

'Tulane University, School of Public Health and Tropical Medicine, 1440 Canal Street, Suite 2200, New Orleans, LA 70112, USA

${ }^{2}$ Department of Sociology, Universiteit Gent, Korte Meer 3, 9000, Gent, Belgium
}

\section{Biomed Central}

(c) 2013 Meekers et al.; licensee BioMed Central Ltd. This is an Open Access article distributed under the terms of the Creative Commons Attribution License (http://creativecommons.org/licenses/by/2.0), which permits unrestricted use, distribution, and reproduction in any medium, provided the original work is properly cited. effects of interpersonal violence on mental health in developed countries [10-15] studies on developing countries are scarce $[16,17]$. This paper analyzes survey data from Bolivia to examine the relationship between women's experiences with intimate partner violence and mental health.

\section{Background}

Definition and prevalence of intimate partner violence Existing studies on IPV frequently use the terms intimate partner violence, domestic abuse, domestic violence, and spousal violence interchangeably $[7,18]$. Spousal violence may refer to partner violence between married couples, 
cohabiting couples, and individuals in other forms of nonmarital unions. Several definitions of domestic violence make specific reference to psychological abuse and threats of harm. The 1993 UN General Assembly defines genderbased violence as any act "that results in, or is likely to result in, physical, sexual, or psychological harm or suffering to women, including threats of such acts, coercion, or arbitrary deprivations of liberty, whether occurring in public or private life" [19]. WHO defines domestic violence as "any behavior within an intimate relationship that causes physical, psychological or sexual harm" [20].

While intimate partner violence exists in all societies, its prevalence varies widely by region $[4,7,20,21]$. WHO surveys show that the percentage of ever-partnered women who reported ever experiencing either physical or sexual violence ranged from $15 \%$ in some Japanese sites to over $60 \%$ in sites in Bangladesh, Ethiopia, and Peru $[4,20]$. Several studies show high rates of IPV in Latin America. For example, $49 \%$ of Peruvian women experienced severe physical violence in their lifetime, and $38 \%$ of women in Colombia report being physically or sexually abused by a recent partner $[4,5]$. A national survey of Paraguay shows that $18.4 \%$ of women who were ever in union experienced psychological IPV, 6.7\% physical IPV, and 3.2\% sexual IPV [16]. In Bolivia, one study found that $52 \%$ and $14 \%$ of women experienced physical and sexual violence, respectively [7], and another found that $40 \%$ had been physically or sexually abused by their spouse in the past year [22]. A recent study of the Tsimane forager-horticulturalists reports that $85 \%$ of women had experienced physical spousal abuse, and 38\% experienced physical abuse in the last year [23].

The WHO surveys also asked women whether their partner had engaged in emotional abuse (e.g., insulting them, belittling or humiliating them, intimidating or scaring them, or threatening to harm them). The percentage of ever-partnered women who reported ever experiencing any such acts ranged from less than $20 \%$ in Samoan sites to $75 \%$ in Ethiopian sites. The percentage who experienced such events during the year before the survey ranged from $12 \%$ in Samoa and urban Serbia and Montenegro to $58 \%$ in Ethiopia [4,20]. The same study also revealed large variations in the percentage of everpartnered women who reported that their partner had put constraints on their physical or social mobility $(21 \%$ in urban Japan compared to nearly $90 \%$ in urban Tanzania).

Low education and socioeconomic status, being younger, and non-marital cohabitation tend to increase women's risk of experiencing domestic violence [7,16,24-27]. Exposure to violence in the parental household - e.g. physical abuse in childhood and witnessing inter-parental abuse - also increases the risk of experiencing spousal abuse in adulthood [28-31] [16]. By contrast, differences in age and education between partners typically have weak or inconsistent associations with women's risk of domestic violence $[7,24,27,32]$.

This study examines the association between various aspects of intimate partner violence and indicators of mental health using recent survey data from Bolivia.

\section{Data}

Our analysis is based on the 2008 Bolivia Demographic and Health Survey [33] which contains data on a nationally representative sample of women aged 15-49 years. The BDHS is part of the larger MEASURE Evaluation Demographic and Health Surveys Phase II project, which is funded by the United States Agency for International Development (USAID). All DHS data are publicly available (www.measuredhs.com/data). The BDHS data were collected by the Bolivian Ministry of Health and Sports (Ministerio de Salud y Deportes) and National Institute for Statistics (Instituto Nacional de Estadística) with technical assistance from ORC Macro (ORC Macro has since become ICF International). The Institutional Review Board of ORC Macro reviewed and approved the MEASURE Demographic and Health Surveys Project Phase II, including the BDHS. The Institutional Review Board of ORC Macro complied with the United States Department of Health and Human Services regulations for the protection of human research subjects (45 CFR 46). Further details about the data collection are available in the survey report [33].

Informed consent was obtained from all respondents. All survey staff received special training for the implementation of the domestic violence module. During the interviews, informed consent was re-iterated before the start of the domestic violence module. Our analysis is restricted to women who are currently married or in union $(\mathrm{n}=10,188)$. After eliminating cases with missing values, 10,119 respondents were retained for analysis. The BDHS questionnaire collected information on a wide range of topics, including domestic violence and mental health. (The survey questionnaire is available at http://www.measuredhs.com/publications/publicationFR228-DHS-Final-Reports.cfm).

\section{Measures}

The BDHS questionnaire includes nine mental health questions, seven of which are a subset of the Self Reporting Questionnaire 20 (SRQ-20), a mental health screening tool developed by WHO [34]. The SRQ-20 has been used widely to screen for the presence of nonpsychotic mental health disorders [35-41]. The SRQ-20 has also been validated as a screening tool for psychological morbidity [42-45]. The BDHS also includes selected mental health questions from the extended version of the Self-Reported Questionnaire, the SRQ-25. 
The SRQ-25 includes four additional questions aimed at identifying probable psychosis and one question about psychogenic non-epileptic seizures, or convulsions. The SRQ-25 has also been widely used to assess the presence of mental health disorders [46-49].

The nine mental health questions in the BDHS aim to help identify four aspects of mental health: depression, anxiety, psychogenic seizures, and psychotic disorders. Since it has been reported that depression and anxiety account for most of the IPV disease burden [50], our study distinguishes between the four different aspects of mental health. To measure whether the respondent has symptoms of depression, all respondents were asked whether they feel tired all the time, whether they cry easily, whether they have difficulty performing daily activities, and whether they find it difficult to make decisions. To identify whether the respondent has symptoms of anxiety disorders, all respondents were asked whether they often have headaches of great intensity at the nape of the neck, whether they feel fear for no apparent reason, and whether they are easily frightened. In addition, one question was used to assess whether the respondent had symptoms of psychotic disorders ("Do you hear voices that talk to you and that others do not hear?") and one question aimed to identify whether the respondent had symptoms of psychogenic non-epileptic seizures ("Have you experienced convulsions or attacks with tongue-biting and loss of consciousness?).

The domestic violence module in the BDHS questionnaire is based on the Conflict Tactics Scale (CTS) developed by Murray Straus [30,51]. Specifically, women were asked whether their current partner engaged in any acts of physical, sexual, or psychological abuse towards them during the 12 months before the survey. To measure exposure to physical abuse, women were asked how often (one time, a few times, or often) in the last 12 months their partner had pushed or pinched them, beaten or kicked them, beaten them with an object, or tried to strangle or burn them. Exposure to sexual abuse was measured by asking women whether their partner forced them to have sexual relations against their will during the year before the survey. Exposure to psychological abuse was measured by asking the respondents how often their partner, in the past 12 months, had accused them of being unfaithful, had been jealous after she talked with a man, had attempted to limit her contact with her family, had humiliated or insulted her, had threatened to abandon her, had threatened to take away her children, had threatened to take away economic support, or had broken things inside the house.

Our summary indicator of experience with intimate partner violence is a 4-category variable that identifies which of the three types of abuse the respondent experienced in the past year (1. No intimate partner abuse; 2 . psychological violence only; 3 . physical abuse or both physical and psychological abuse; 4 . any sexual abuse).

Our control variables include the respondent's socioeconomic and demographic status, her partner's socioeconomic and demographic status, and exposure to domestic abuse in the respondent's parental household. Respondent characteristics include her age group, place of residence (rural vs. urban), marital status (married vs. cohabiting), region of residence, employment status, and socioeconomic status. The respondent's socioeconomic status was assessed using the DHS wealth index, which uses information on household assets, services and amenities to classify respondents into wealth quintiles [52].

Controls for the partner's characteristics include his age group, the age difference between the respondent and her partner, partner's level of education, and the difference in the level of education between the respondent and her partner. To measure the respondent's exposure to domestic abuse in her parental household, we include dichotomous variables that measure whether she was physically abused in childhood (yes/no), whether she was psychologically abused as a child (yes/no), and whether her father beat her mother (no vs. yes/don't know). Women who reported being punished as a child by being beaten, slapped, having their ears pulled, having food withheld, or having water thrown on them were coded as having experienced physical abuse. Women who reported punishment by being shouted at, insulted, locked up, left outside, having their clothes taken away, or being ignored for more than a day were coded as having experienced psychological abuse in childhood.

\section{Methods}

We use probit regression models to examine the association between exposure to intimate partner violence and indicators of mental health, after controlling for other factors [53]. To facilitate interpretation, the probit results are converted to the predicted probability of experiencing various mental health outcomes using STATA12's margins command [54]. For simplicity, we present the predicted probabilities in the form of the predicted percentage of women who experienced each mental health outcome. For each health outcome, we present two models. The first model shows the unadjusted percentage of respondents who experienced the outcome; the second model shows the adjusted percentages after controlling for other factors. In the text, we will focus on the IPV variables and childhood experience with violence. Because we cannot rule out that some women have left a relationship because of abuse, our analysis may underestimate the association between intimate partner violence on mental health. 


\section{Results}

Table 1 describes the characteristics of the working sample of married and cohabiting women. Most women lived in urban areas (61.1\%), but had a relatively low level of education; less than half of women attended secondary school. As anticipated, the sample has a fairly young age distribution. Nearly two thirds of women are married (62.8\%), while the remainder are cohabiting.

Nearly half of all women (44.3\%) reported that their partner was one to five years older than themselves, while $26.7 \%$ reported that their partner was at least six years older. On average, men have somewhat higher levels of education than women. In over half of the couples (55.0\%), the male partner has the highest level of education. Nevertheless, one in five women (21.6\%) report having a higher level of education than their partner.

The third panel of Table 1 shows information about domestic abuse in the respondent's parental household. Overall, $71.7 \%$ of women experienced physical abuse as a child, and $42.4 \%$ experienced psychological abuse. When respondents were asked whether their father beat their mother, only $46.1 \%$ denied that this happened. The rest either admitted that their father beat their mother or reported not knowing whether this happened.

Overall, $47.2 \%$ of women reported experiencing some type of intimate partner violence in the past year: $21.1 \%$ experienced only psychological abuse, and 19.2\% experienced both physical abuse or both physical and psychological abuse. In addition, 6.9\% were sexually abused by their partner. Most victims of sexual abuse by their partner were also physically or psychologically abused (82.8\% and $94.8 \%$, respectively, not shown).

Tables 2 and 3 show the predicted percentage of women who reported having experienced various physical and mental health outcomes, by type of intimate partner violence experienced. Table 2 shows indicators of depression; Table 3 shows indicators anxiety, psychotic disorders, and psychogenic non-epileptic seizures. For both tables, two models are shown for each indicator. The first model shows the unadjusted percentage of women who report experiencing the symptom; the second model shows the adjusted percentage after controlling for other factors.

\section{Depression}

The unadjusted results shown in Table 2 indicate that the percentage of women who report having symptoms of depression varies according to their exposure to intimate partner violence. The adjusted percentages show that these differences persist after controlling for other factors. After controls, the percentage of women who report feeling tired all the time remains significantly higher among women experienced psychological abuse only (57.5\%; $\mathrm{p}<.001)$, who experienced physical abuse $(55.5 \%$; $\mathrm{p}<.01)$, and those who experienced sexual abuse $(66.4 \%$; $<.001)$.
The results for 'difficulty doing daily activities' and 'difficulty making decisions' show nearly identical patterns. That is, psychological abuse and physical abuse have nearly equal effects, while sexual abuse has the strongest effect. The results further suggest that women who were physically or sexually abused by their partner are much more likely than unabused women to report that they cry easily $(78.6 \%$ and $85.9 \%$, respectively, vs. $72.7 \%$; $\mathrm{p}<.001)$. The adjusted percentages show that these effects cannot be explained by the respondent's socioeconomic status, her relative age and education compared to her partner, or by experiences with abuse in her parental household.

Childhood experience with abuse in the parental household is also associated with the risk the women's report experiencing symptoms of depression, but the effects are not consistent. Women who report experiencing physical abuse in childhood are more likely than other women to report crying easily $(76.2 \%$ vs. $72.1 \%$; $\mathrm{p}<.001)$. However, experiencing physical abuse in childhood is not associated with other symptoms of depression. In addition, women who report that their father beat their mother, or who report they were unsure about it, are more likely than other women to cry easily $(76.7 \%$ vs. $73.2 \% ; \mathrm{p}<.01$ ) and more likely to have difficulty making decisions $(50.1 \%$ vs. $44.1 \%$; $\mathrm{p}<.001)$.

\section{Anxiety}

The prevalence of symptoms of anxiety varies depending on the respondent's exposure to domestic abuse in the past year. For example, after controls, $46.0 \%$ of women who report not being abused by their partner report having feelings of fear without apparent reason. This percentage increased to $49.7 \%(\mathrm{p}<.05)$ for those women who experienced psychological abuse and to 53.5\% $(\mathrm{p}<.001)$ for those who experienced physical abuse. Among women who were sexually abused, 59.3\% express having feelings of fear $(\mathrm{p}<.001)$. It is noteworthy that psychological abuse and physical abuse by a partner are both associated with significantly higher scores on all three anxiety indicators. However, psychological and physical abuse have nearly identical effects on 'scaring easily' and 'having headaches of great intensity.' By contrast, physical abuse has a stronger effect than psychological abuse on the likelihood that women have feelings of fear for no apparent reason. Women who were sexually abused by their partner have the highest scores on all three indicators of anxiety.

Childhood exposure to domestic violence is not associated with having symptoms of anxiety. Although women who were physically abused in childhood are slightly more likely than other women to report having headaches of great intensity $(56.1 \%$ vs. $52.1 \%$; $<<.001)$, there is no effect on the other two indicators of anxiety. 


\section{Table 1 Sample description (married and cohabiting women only)}

$\%$ (weighted) $\%$ (unweighted)

Residence

Urban

Rural

Education

None

Primary

Secondary

Higher

SES

First quintile

Second quintile

Third quintile

Fourth quintile

Fifth quintile

Respondent's work status

$$
\begin{aligned}
& \text { Working } \\
& \text { Not working }
\end{aligned}
$$

Region

La Paz
Chuquisaca
Cochabamba
Oruro
Potosí
Tarija
Santa Cruz
Beni
Pando

Respondent's age

$$
\text { 15-24 }
$$

25-34

$35-44$

45-49

Respondent's marital status

Married

Cohabiting

Partner's age

$14-24$
$25-34$
$35-44$
$45+$

Partner's age, relative to respondent

$$
\begin{aligned}
& \text { Same age or younger } \\
& 1-5 \text { years older }
\end{aligned}
$$

$61.1 \% \quad 58.5 \% \quad 5,924$

38.9

6.4$$
51.4
$$

14.0

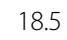

6.2

50.5

28.0

15.3

20.3

19.5

19.9

20.8

19.4

64.5

35.5

18.7

8.7

13.0

8.4

10.6

9.8

18.8

7.1

4.8

6,178

3,941

1,029

3,439

3,353

2,290

$41.5 \quad 4,195$

Table 1 Sample description (married and cohabiting women only) (Continued)

\begin{tabular}{lccr}
\hline $\begin{array}{l}\text { 6 or more years older } \\
\text { Partner's education }\end{array}$ & 26.7 & 27.6 & 2,795 \\
$\quad$ None & 1.5 & 1.8 & 181 \\
Primary & 44.0 & 44.5 & 4,502 \\
$\quad$ Secondary & 35.9 & 33.9 & 3,429 \\
$\quad$ Higher & 18.5 & 19.8 & 1,997 \\
$\begin{array}{l}\text { Education differences } \\
\text { between partners }\end{array}$ & & & \\
$\quad$ Wife is more educated & 21.6 & 23.2 & 5,348 \\
$\quad$ Same level of education & 23.4 & 24.0 & 2,425 \\
$\quad$ Husband is more educated & 55.0 & 52.8 & 2,334 \\
$\begin{array}{l}\text { Respondent experienced } \\
\text { physical abuse in childhood }\end{array}$ & & & \\
No & & & \\
Yes & 28.3 & 29.0 & 2,930 \\
\end{tabular}

Respondent experienced

psychological abuse in childhood

$\begin{array}{llll}\text { No } & 57.6 & 59.7 & 6,033 \\ \text { Yes } & 42.4 & 40.4 & 4,081\end{array}$

Respondent reports father beat mother

$\begin{array}{llll}\text { No } & 46.1 & 48.1 & 5,250 \\ \text { Yes/Don't know } & 53.9 & 51.9 & 4,860\end{array}$

Respondent's experience

with intimate partner

violence in last 12 months

\begin{tabular}{lccr} 
None & 52.8 & 53.0 & 5,365 \\
Psychological abuse only & 21.1 & 20.8 & 2,106 \\
Physical abuse (no sexual) & 19.2 & 19.1 & 1,933 \\
Sexual abuse $\Psi$ & 6.9 & 7.1 & 715 \\
Total & $100 \%$ & $100 \%$ & 10,119 \\
\hline
\end{tabular}

$\psi$ Most victims of sexual abuse also experienced psychological and/or physical abuse.

Psychological abuse in childhood, or having witnessed spousal abuse in the parental household are not associated with any of the three symptoms of anxiety.

\section{Psychogenic non-epileptic seizures}

The percentage of women who reported having convulsions or attacks with tongue biting is associated with intimate partner violence. The adjusted percentage of women who report having convulsions or attacks with tongue biting is highest among women who experienced sexual abuse $(12.4 \% ; \mathrm{p}<.001)$, followed by physical abuse $(9.4 \% ; \mathrm{p}<.001)$ and psychological abuse $(8.6 \% ; \mathrm{p}<.01)$. Having experienced physical or psychological abuse in childhood is not associated with the likelihood that women report having had convulsions. Three regions 
Table 2 Unadjusted and adjusted percentage of women experiencing various symptoms of depression, by exposure to intimate partner violence, socioeconomic and demographic characteristics of the respondent, partner's characteristics, and exposure to domestic abuse in the parental household

\begin{tabular}{|c|c|c|c|c|c|c|c|c|}
\hline & \multicolumn{8}{|c|}{ Symptoms of depression } \\
\hline & \multicolumn{2}{|l|}{$\begin{array}{l}\text { Feels tired all } \\
\text { the time }\end{array}$} & \multicolumn{2}{|l|}{ Cries easily } & \multicolumn{2}{|c|}{$\begin{array}{l}\text { Difficulty doing } \\
\text { daily activities }\end{array}$} & \multicolumn{2}{|l|}{$\begin{array}{l}\text { Difficulty } \\
\text { making } \\
\text { decisions }\end{array}$} \\
\hline & (1) & $(2)$ & (1) & $(2)$ & (1) & $(2)$ & $(\mathbf{1})$ & $(2)$ \\
\hline & Unadjusted & Adjusted & Unadjusted & Adjusted & Unadjusted & Adjusted & Unadjusted & Adjusted \\
\hline & $\begin{array}{l}\text { Percentage } \\
\text { (Cl) }\end{array}$ & $\begin{array}{l}\text { Percentage } \\
\text { (Cl) }\end{array}$ & $\begin{array}{l}\text { Percentage } \\
\text { (CI) }\end{array}$ & $\begin{array}{l}\text { Percentage } \\
\text { (Cl) }\end{array}$ & $\begin{array}{l}\text { Percentage } \\
\text { (Cl) }\end{array}$ & $\begin{array}{l}\text { Percentage } \\
\text { (Cl) }\end{array}$ & $\begin{array}{l}\text { Percentage } \\
\text { (Cl) }\end{array}$ & $\begin{array}{l}\text { Percentage } \\
\text { (Cl) }\end{array}$ \\
\hline \multicolumn{9}{|l|}{ Intimate partner violence } \\
\hline \multirow[t]{2}{*}{ No abuse (reference) } & 49.8 & 49.9 & 72.1 & 72.7 & 28.0 & 28.0 & 42.1 & 42.7 \\
\hline & $(48.1-51.6)$ & $(48.2-51.6)$ & $(70.6-73.7)$ & $(71.2-74.2)$ & $(26.3-29.7)$ & $(26.4-29.7)$ & $(40.3-43.8)$ & $(41.0-44.4)$ \\
\hline \multirow[t]{2}{*}{ Psychol. abuse only } & $55.9^{* * *}$ & $57.5^{* * *}$ & 74.6 & 74.2 & $33.6^{* * *}$ & $34.0^{* * *}$ & $50.8^{* * *}$ & $50.8^{* * *}$ \\
\hline & $(53.6-58.3)$ & $(55.2-59.8)$ & $(72.2-77.0)$ & $(71.8-76.6)$ & $(31.3-35.9)$ & $(31.7-36.3)$ & $(48.2-53.3)$ & $(48.2-53.4)$ \\
\hline \multirow[t]{2}{*}{ Physical abuse (no sexual) } & $56.3^{* * *}$ & $55.5^{* *}$ & $79.4^{* * *}$ & $78.6^{* * *}$ & $34.1^{* * *}$ & $34.1^{* * *}$ & $51.9^{* * *}$ & $50.7^{* * *}$ \\
\hline & $(53.6-58.9)$ & $(52.9-58.1)$ & $(77.1-81.7)$ & $(76.3-80.9)$ & $(31.4-36.7)$ & $(31.4-36.8)$ & $(49.1-54.7)$ & $(47.9-53.6)$ \\
\hline \multirow[t]{2}{*}{ Sexual abuse $¥$} & $68.3^{* * *}$ & $66.4^{* * *}$ & $86.3^{* * *}$ & $85.9^{* * *}$ & $43.8^{* * *}$ & $42.4^{* * *}$ & $63.8^{* * *}$ & $62.4^{* * *}$ \\
\hline & $(64.0-72.5)$ & $(62.2-70.6)$ & (83.1-89.6) & (82.4-89.3) & $(39.0-48.7)$ & $(37.6-47.1)$ & $(59.5-68.2)$ & $(58.2-66.6)$ \\
\hline \multicolumn{9}{|l|}{$\begin{array}{l}\text { Respondent experienced } \\
\text { physical abuse in childhood }\end{array}$} \\
\hline \multirow[t]{2}{*}{ No (reference) } & & 52.3 & & 72.1 & & 31.0 & & 45.9 \\
\hline & & $(50.1-54.5)$ & & $(70.1-74.0)$ & & $(28.7-33.3)$ & & $(43.5-48.3)$ \\
\hline \multirow[t]{2}{*}{ Yes } & & 54.3 & & $76.2^{* * *}$ & & 31.6 & & 47.9 \\
\hline & & $(52.9-55.7)$ & & $(75.0-77.5)$ & & $(30.2-33.1)$ & & $(46.4-49.4)$ \\
\hline \multicolumn{9}{|l|}{$\begin{array}{l}\text { Respondent experienced } \\
\text { psychological abuse in } \\
\text { childhood }\end{array}$} \\
\hline \multirow[t]{2}{*}{ No (reference) } & & 55.4 & & 75.7 & & 31.9 & & 48.7 \\
\hline & & $(54.0-56.9)$ & & $(74.3-77.1)$ & & $(30.2-33.6)$ & & $(47.0-50.3)$ \\
\hline \multirow[t]{2}{*}{ Yes } & & $51.4^{* *}$ & & 74.1 & & 30.8 & & $45.6^{*}$ \\
\hline & & $(49.5-53.4)$ & & $(72.4-75.8)$ & & $(29.0-32.7)$ & & $(43.5-47.6)$ \\
\hline \multicolumn{9}{|l|}{$\begin{array}{l}\text { Respondent reports father } \\
\text { beat mother }\end{array}$} \\
\hline \multirow[t]{2}{*}{ No (reference) } & & 53.3 & & 73.2 & & 30.9 & & 44.1 \\
\hline & & $(51.5-55.1)$ & & $(71.6-74.8)$ & & $(29.3-32.5)$ & & $(42.3-45.9)$ \\
\hline \multirow[t]{2}{*}{ Yes/Don't know } & & 54.1 & & $76.7^{* *}$ & & 31.9 & & $50.1^{* * *}$ \\
\hline & & $(52.4-55.8)$ & & (75.3-78.0) & & $(30.2-33.6)$ & & $(48.4-51.8)$ \\
\hline \multicolumn{9}{|l|}{ Region } \\
\hline \multirow[t]{2}{*}{ La Paz (reference) } & & 62.3 & & 78.9 & & 34.6 & & 51.8 \\
\hline & & $(59.8-64.7)$ & & $(76.6-81.2)$ & & $(31.9-37.4)$ & & $(49.3-54.3)$ \\
\hline \multirow[t]{2}{*}{ Chuquisaca } & & $44.8^{* * *}$ & & 80.2 & & $24.8^{* * *}$ & & 48.3 \\
\hline & & $(39.6-50.0)$ & & (77.3-83.0) & & $(20.6-29.0)$ & & $(44.3-52.3)$ \\
\hline \multirow[t]{2}{*}{ Cochabamba } & & $57.8^{*}$ & & 77.0 & & 35.6 & & $47.0^{*}$ \\
\hline & & $(54.8-60.9)$ & & $(74.7-79.4)$ & & $(32.3-39.0)$ & & $(43.7-50.4)$ \\
\hline \multirow[t]{2}{*}{ Oruro } & & $49.0^{* * *}$ & & $73.5^{* *}$ & & $27.2^{* *}$ & & $47.3^{*}$ \\
\hline & & $(45.3-52.6)$ & & $(70.2-76.8)$ & & $(23.5-31.0)$ & & $(43.5-51.0)$ \\
\hline \multirow[t]{2}{*}{ Potosí } & & $54.5^{* * *}$ & & $65.3^{* * *}$ & & $27.4^{* *}$ & & $43.8^{* *}$ \\
\hline & & $(51.0-57.9)$ & & $(61.6-68.9)$ & & $(23.6-31.3)$ & & $(38.5-49.1)$ \\
\hline
\end{tabular}


Table 2 Unadjusted and adjusted percentage of women experiencing various symptoms of depression, by exposure to intimate partner violence, socioeconomic and demographic characteristics of the respondent, partner's characteristics, and exposure to domestic abuse in the parental household (Continued)

\begin{tabular}{|c|c|c|c|c|}
\hline \multirow[t]{2}{*}{ Tarija } & $53.1^{* * *}$ & $73.7^{*}$ & 38.1 & 55.2 \\
\hline & $(49.6-56.6)$ & $(70.2-77.2)$ & $(34.9-41.3)$ & $(51.6-58.7)$ \\
\hline \multirow[t]{2}{*}{ Santa Cruz } & $45.9^{* * *}$ & $73.2^{* *}$ & $28.8^{* *}$ & $44.0^{* * *}$ \\
\hline & $(43.1-48.7)$ & (70.6-75.9) & $(26.3-31.4)$ & $(41.8-46.2)$ \\
\hline \multirow[t]{2}{*}{ Beni } & $33.9^{* * *}$ & $70.5^{* * *}$ & $21.3^{* * *}$ & $32.3^{* * *}$ \\
\hline & $(28.1-39.6)$ & $(66.8-74.1)$ & $(17.5-25.1)$ & (27.0-37.6) \\
\hline \multirow[t]{2}{*}{ Pando } & $54.2^{* *}$ & $66.8^{* * *}$ & 31.3 & 46.5 \\
\hline & $(48.8-59.5)$ & $(61.9-71.8)$ & $(26.4-36.2)$ & $(39.4-53.5)$ \\
\hline \multicolumn{5}{|l|}{ Residence } \\
\hline \multirow[t]{2}{*}{ Urban (reference) } & 54.6 & 75.1 & 31.5 & 47.4 \\
\hline & $(52.5-56.6)$ & $(73.4-76.9)$ & $(29.4-33.5)$ & $(45.3-49.4)$ \\
\hline \multirow[t]{2}{*}{ Rural } & 52.4 & 74.8 & 31.4 & 47.3 \\
\hline & $(49.5-55.3)$ & $(72.5-77.2)$ & $(28.5-34.3)$ & $(46.2-50.4)$ \\
\hline \multicolumn{5}{|l|}{ Education } \\
\hline \multirow[t]{2}{*}{ None (reference) } & 57.7 & 76.2 & 34.0 & 45.3 \\
\hline & $(52.1-63.3)$ & (71.3-81.2) & $(28.9-39.2)$ & $(39.6-51.0)$ \\
\hline \multirow[t]{2}{*}{ Primary } & 57.8 & 74.4 & 33.5 & 49.7 \\
\hline & $(55.7-59.9)$ & (72.5-76.3) & $(31.6-35.5)$ & $(44.1-48.9)$ \\
\hline \multirow[t]{2}{*}{ Secondary } & $50.2^{*}$ & 77.2 & 28.9 & 47.7 \\
\hline & $(47.6-52.9)$ & (74.9-79.4) & $(26.4-31.4)$ & $(45.0-50.4)$ \\
\hline \multirow[t]{2}{*}{ Higher } & $44.1^{* *}$ & 72.4 & 26.9 & 38.3 \\
\hline & (39.4-48.9) & $(68.2-76.7)$ & $(22.8-31.0)$ & $(33.5-43.1)$ \\
\hline \multicolumn{5}{|l|}{ SES } \\
\hline \multirow[t]{2}{*}{ First quintile (reference) } & 57.4 & 72.6 & 30.4 & 45.1 \\
\hline & $(53.1-61.7)$ & $(69.2-76.1)$ & $(26.6-34.2)$ & $(41.0-49.2)$ \\
\hline \multirow[t]{2}{*}{ Second quintile } & 55.2 & 74.4 & 34.1 & 46.6 \\
\hline & $(51.7-58.8)$ & $(71.6-77.3)$ & $(30.8-37.3)$ & $(43.6-49.7)$ \\
\hline \multirow[t]{2}{*}{ Third quintile } & 55.7 & 75.9 & 35.3 & 50.0 \\
\hline & $(53.0-58.3)$ & $(73.6-78.3)$ & $(32.4-38.1)$ & $(47.3-52.6)$ \\
\hline \multirow[t]{2}{*}{ Fourth quintile } & 53.1 & $78.4^{*}$ & 33.0 & 50.6 \\
\hline & $(50.2-56.1)$ & $(75.8-80.9)$ & $(30.0-36.0)$ & $(47.5-53.8)$ \\
\hline \multirow[t]{2}{*}{ Fifth quintile } & $47.4^{* *}$ & 73.2 & $23.6^{*}$ & 43.4 \\
\hline & $(43.5-51.2)$ & $(69.9-76.6)$ & $(20.2-26.9)$ & $(39.9-47.0)$ \\
\hline \multicolumn{5}{|l|}{ Work status } \\
\hline \multirow[t]{2}{*}{ Not working (reference) } & 51.6 & 75.1 & 29.8 & 46.5 \\
\hline & $(49.5-53.7)$ & $(73.3-76.9)$ & $(27.8-31.8)$ & $(44.3-48.6)$ \\
\hline \multirow[t]{2}{*}{ Working } & 54.8 & 75.0 & 32.3 & 47.8 \\
\hline & $(53.3-56.3)$ & $(73.6-76.3)$ & $(30.7-33.8)$ & $(46.3-49.3)$ \\
\hline \multicolumn{5}{|l|}{ Age } \\
\hline \multirow[t]{2}{*}{ 15-24 (reference) } & 48.1 & 72.0 & 27.9 & 49.2 \\
\hline & $(43.8-52.3)$ & $(67.7-76.2)$ & $(23.9-32.0)$ & $(44.6-53.8)$ \\
\hline \multirow[t]{2}{*}{$25-34$} & 52.1 & 74.5 & 30.3 & 46.5 \\
\hline & $(50.0-54.3)$ & $(72.5-76.6)$ & $(28.2-32.5)$ & $(44.1-48.9)$ \\
\hline
\end{tabular}


Table 2 Unadjusted and adjusted percentage of women experiencing various symptoms of depression, by exposure to intimate partner violence, socioeconomic and demographic characteristics of the respondent, partner's characteristics, and exposure to domestic abuse in the parental household (Continued)

\begin{tabular}{|c|c|c|c|c|}
\hline \multirow[t]{2}{*}{$35-44$} & $56.6^{* *}$ & 75.8 & 32.7 & 46.3 \\
\hline & $(53.9-59.3)$ & $(73.4-78.1)$ & $(30.0-35.3)$ & $(43.6-49.1)$ \\
\hline \multirow[t]{2}{*}{$45-49$} & $59.5^{* *}$ & 78.5 & $36.4^{*}$ & 50.0 \\
\hline & $(55.0-63.9)$ & $(74.5-82.5)$ & $(31.6-41.1)$ & $(44.8-55.3)$ \\
\hline \multicolumn{5}{|l|}{ Marital status } \\
\hline \multirow[t]{2}{*}{ Married (reference) } & 54.8 & 75.2 & 30.9 & 46.8 \\
\hline & $(53.3-56.4)$ & $(73.8-76.7)$ & $(29.4-32.5)$ & $(45.2-48.4)$ \\
\hline \multirow[t]{2}{*}{ Cohabiting } & $51.9^{*}$ & 75.0 & 32.4 & 48.3 \\
\hline & $(49.8-54.1)$ & $(72.5-76.7)$ & $(30.3-34.4)$ & $(46.2-50.4)$ \\
\hline \multicolumn{5}{|l|}{ Partner's age } \\
\hline \multirow[t]{2}{*}{ 14-24 (reference) } & 54.1 & 76.6 & 32.4 & 48.0 \\
\hline & $(48.7-59.5)$ & $(72.2-80.9)$ & $(27.4-37.4)$ & $(42.4-53.6)$ \\
\hline \multirow[t]{2}{*}{$25-34$} & 54.0 & 76.2 & 28.4 & 46.2 \\
\hline & $(51.4-56.6)$ & $(74.0-78.5)$ & $(25.8-31.0)$ & $(43.5-49.0)$ \\
\hline \multirow[t]{2}{*}{$35-44$} & 53.5 & 74.0 & 31.7 & 48.4 \\
\hline & $(51.4-55.7)$ & $(71.9-76.1)$ & $(29.6-33.9)$ & $(46.0-50.8)$ \\
\hline \multirow[t]{2}{*}{$45+$} & 53.5 & 73.9 & 35.2 & 47.1 \\
\hline & $(49.6-57.4)$ & $(70.2-77.7)$ & $(31.4-39.0)$ & $(42.9-51.3)$ \\
\hline \multicolumn{5}{|l|}{$\begin{array}{l}\text { Partner's age, relative to } \\
\text { respondent }\end{array}$} \\
\hline \multirow[t]{2}{*}{ Same age or younger (ref.) } & 55.4 & 76.7 & 33.2 & 47.7 \\
\hline & $(52.8-58.0)$ & $(74.5-78.8)$ & $(30.8-35.6)$ & $(45.2-50.2)$ \\
\hline \multirow[t]{2}{*}{$1-5$ yrs. older } & 53.1 & 74.2 & 31.2 & 47.2 \\
\hline & $(51.4-54.7)$ & $(72.5-75.8)$ & $(29.5-33.0)$ & $(45.4-49.0)$ \\
\hline \multirow[t]{2}{*}{$6+$ yrs. older } & 53.1 & 74.7 & 29.9 & 47.1 \\
\hline & $(50.4-55.7)$ & $(72.4-77.0)$ & $(27.4-32.4)$ & $(44.5-49.8)$ \\
\hline \multicolumn{5}{|l|}{ Partner's education } \\
\hline \multirow[t]{2}{*}{ None (reference) } & 52.6 & 75.4 & 20.3 & 41.7 \\
\hline & $(42.9-62.4)$ & $(67.8-82.9)$ & $(12.5-28.0)$ & $(32.4-51.0)$ \\
\hline \multirow[t]{2}{*}{ Primary } & 55.7 & 75.4 & $32.0^{* *}$ & 47.1 \\
\hline & $(53.3-58.1)$ & $(73.3-77.5)$ & $(29.9-34.2)$ & $(44.7-49.6)$ \\
\hline \multirow[t]{2}{*}{ Secondary } & 55.0 & 75.2 & $32.1^{*}$ & 49.3 \\
\hline & $(52.9-57.2)$ & $(73.2-77.3)$ & $(30.2-34.0)$ & $(47.2-51.5)$ \\
\hline \multirow[t]{2}{*}{ Higher } & 46.6 & 73.8 & 29.6 & 44.3 \\
\hline & $(42.5-50.6)$ & $(70.4-77.2)$ & $(26.0-33.2)$ & $(40.3-48.3)$ \\
\hline \multicolumn{5}{|l|}{$\begin{array}{l}\text { Education difference } \\
\text { between partners }\end{array}$} \\
\hline \multirow[t]{2}{*}{ Wife is more educated (ref.) } & 53.5 & 73.9 & 31.1 & 46.5 \\
\hline & $(50.7-56.3)$ & $(71.2-76.5)$ & $(28.4-33.8)$ & $(43.4-49.5)$ \\
\hline \multirow[t]{2}{*}{ Same level of education } & 50.3 & 73.9 & 29.0 & 46.1 \\
\hline & $(47.7-52.9)$ & $(71.4-76.4)$ & $(26.7-31.2)$ & $(43.7-48.6)$ \\
\hline \multirow[t]{2}{*}{ Husband is more educated } & 55.3 & 73.8 & 32.6 & 48.2 \\
\hline & $(53.4-57.2)$ & $(74.3-77.6)$ & $(30.7-34.5)$ & $(46.2-50.1)$ \\
\hline
\end{tabular}

${ }^{*} p<0.05 * * 0.0 .01{ }^{* * *} p<0.001$.

$\psi$ Respondents who experienced sexual abuse may also have experienced psychological and/or physical abuse. 
stand out because of their high prevalence of convulsions: Cochabamba (11.3\%), Beni (11.6\%), and Pando (12.9\%).

\section{Psychotic disorders}

Intimate partner violence is associated with hearing voices that others do not hear. The percentage of women who reported hearing voices is significantly higher among women who experienced sexual abuse $(24.0 \%$; $\mathrm{p}<.001)$ or physical abuse $(15.3 \%$; $\mathrm{p}<.01)$ but not among women who experienced only psychological abuse. Women from Tarija and Pando are more likely to report such symptoms.

\section{Discussion}

The results of this study confirm that intimate partner violence is common in Bolivia. These findings are consistent with a large body of literature that shows that intimate partner violence is common in Latin America [1-5]. Our data show that overall, nearly one in two women in Bolivia (47\%) experienced some type of intimate partner violence in the past year. Nineteen percent of women in union report being physically abused by their partner and seven percent were sexually abused by him. Most victims of sexual abuse were also physically and/or psychologically abused. Moreover, one in five Bolivian women (21\%) reported that they were psychologically abused by their partner, although they were not physically or sexually abused. A recent review article on the health implications of IPV noted that the majority of studies also found that women often experience more than one type of IPV [55].

As noted elsewhere, there is a need for further research on the implications of different types of IPV and of experiencing multiple types of IPV [55]. Existing studies on the association between IPV and health outcomes often examine IPV as a single construct, without distinguishing between different types of IPV. The high prevalence of psychological abuse by intimate partners (in absence of either physical or sexual abuse) that we have observed in the Bolivia data highlights the need to study not only the potential adverse effects of different types of IPV on mental health, but to also examine whether experienced only psychological abuse affects mental health. Although numerous studies have examined the relationship between IPV and mental health in the developed world, our study is one of the few populationbased studies that examines this association in a developing country (for other examples, see [16,17]).

Our findings support findings from several other studies - mostly from the U.S. - that show that intimate partner violence is associated not only with physical health, but also with mental health [56-61]. Studies on the association between intimate partner violence and mental health among Latina women in the U.S. indicate that experience with intimate partner violence is associated with adverse mental health effects [62-64]. Our data from Bolivia show that women who experienced intimate partner violence are more likely than other women to experience symptoms of mental health disorders.

While nearly all existing studies examine the combined effects of physical, psychological, and sexual violence on health outcomes, our study distinguishes between these three types of partner abuse. Moreover, we also specifically examined the effect of having experienced only psychological IPV. Because there are indications that the total IPV disease burden is caused mostly by depression and to a lesser extent by anxiety $[50,55]$, our study separately examined the association between different types of IPV and symptoms of depression, anxiety, psychogenic seizures, and psychotic disorders, rather than using a single mental health construct as has been done in some other studies $[16,17]$.

As anticipated, our analyses show that Bolivian women who experienced physical abuse by their intimate partner in the last year are much more likely to experience symptoms of depression, anxiety, psychogenic seizures, and psychotic disorders, irrespective of their childhood experiences with domestic abuse, their social and economic status, or partner and couple characteristics. Women who were sexually abused by an intimate partner are even more likely to experience undesirable mental health outcomes. It is noteworthy that most victims of sexual abuse by their partner were also physically or psychologically abused. This combination of different types of abuse is likely to have exacerbated these negative mental health outcomes. These findings are consistent with other studies of the effect of different types of IPV on symptoms of mental health problems. It also reinforces earlier findings that experiencing multiple types of IPV increases the likelihood of probability of experiencing symptoms of depression and post-traumatic stress disorder [16,55].

Another important finding of our study is that for Bolivian women, experiencing psychological abuse is also associated with an increased risk of experiencing symptoms of depression, anxiety, or psychogenic seizures. In fact, women who only experienced psychological abuse by their intimate partner are nearly as likely as women who were physically abused to report having symptoms of depression, anxiety, and psychogenic seizures. A study of Spanish women also found that psychological IPV had a similar effect on symptoms of depression as physical IPV [65]. Our study did not find any evidence that psychological abuse alone is associated with an increased prevalence of symptoms of psychotic disorders. These findings are consistent with U.S. studies that found that physical, emotional, and sexual intimate partner violence were each associated with mental health problems, including depression $[59,60,66,67]$. 
Table 3 Unadjusted and adjusted percentage of women who experienced various symptoms of anxiety, symptoms of psychogenic non-epileptic seizures, and of psychotic disorders, by exposure to intimate partner violence, socioeconomic and demographic characteristics of the respondent, partner's characteristics, and exposure to domestic abuse in the parental household

\begin{tabular}{|c|c|c|c|c|c|c|c|c|c|c|}
\hline & \multicolumn{6}{|c|}{ Symptoms of anxiety } & \multirow{2}{*}{\multicolumn{2}{|c|}{ 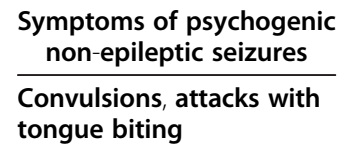 }} & \multirow{2}{*}{\multicolumn{2}{|c|}{$\begin{array}{l}\begin{array}{c}\text { Symptoms of psychotic } \\
\text { disorders }\end{array} \\
\text { Hears voices that others } \\
\text { do not hear }\end{array}$}} \\
\hline & \multicolumn{2}{|c|}{$\begin{array}{l}\text { Feelings of fear for no } \\
\text { apparent reason }\end{array}$} & \multicolumn{2}{|l|}{ Scares easily } & \multicolumn{2}{|c|}{$\begin{array}{l}\text { Headaches of great } \\
\text { intensity }\end{array}$} & & & & \\
\hline & (1) & (2) & (1) & (2) & (1) & (2) & (1) & (2) & (1) & (2) \\
\hline & Unadjusted & Adjusted & Unadjusted & Adjusted & Unadjusted & Adjusted & Unadjusted & Adjusted & Unadjusted & Adjusted \\
\hline & Percentage & Percentage & Percentage & Percentage & Percentage & Percentage & Percentage & Percentage & Percentage & Percentage \\
\hline & (Cl) & (Cl) & $(\mathrm{Cl})$ & (Cl) & $(\mathrm{Cl})$ & $(\mathrm{Cl})$ & (Cl) & (Cl) & (Cl) & $(\mathrm{Cl})$ \\
\hline \multicolumn{11}{|l|}{ Intimate partner violence } \\
\hline \multirow[t]{2}{*}{ No abuse (reference) } & 45.6 & 46.0 & 62.8 & 63.3 & 51.0 & 50.8 & 6.5 & 6.4 & 11.2 & 11.5 \\
\hline & $(43.9-47.3)$ & $(44.3-47.7)$ & $(61.1-64.6)$ & $(61.6-65.0)$ & $(49.3-52.7)$ & $(49.1-52.4)$ & $(5.7-7.4)$ & $(5.6-7.2)$ & $(10.1-12.3)$ & $(10.4-12.7)$ \\
\hline \multirow[t]{2}{*}{ Psychol. abuse only } & $49.0^{*}$ & $49.7^{*}$ & $67.0^{* *}$ & $67.0^{*}$ & $58.5^{* * *}$ & $58.9^{* *}$ & $8.3^{*}$ & $8.6^{* *}$ & $13.9^{*}$ & 13.5 \\
\hline & $(46.4-51.6)$ & $(50.8-56.2)$ & $(64.7-69.4)$ & $(64.6-69.4)$ & $(55.9-61.1)$ & $(56.4-61.5)$ & $(6.7-9.8)$ & $(7.0-10.2)$ & $(12.0-15.7)$ & $(11.7-15.3)$ \\
\hline \multirow[t]{2}{*}{ Physical abuse (no sexual) } & $54.9^{* * *}$ & $53.5^{* * *}$ & $67.6^{* *}$ & 66.6 & $58.0^{* * *}$ & $58.3^{* * *}$ & $9.3^{* *}$ & $9.4^{* * *}$ & $15.7^{* * *}$ & $15.3^{* *}$ \\
\hline & $(52.1-57.7)$ & $(50.8-56.2)$ & $(64.9-70.3)$ & $(63.9-69.4)$ & $(55.3-60.7)$ & $(55.6-60.9)$ & $(7.7-10.8)$ & $(7.9-11.0)$ & $(13.4-17.9)$ & $(13.0-17.5)$ \\
\hline \multirow[t]{2}{*}{ Sexual abuse $¥$} & $61.2^{* * *}$ & $59.3^{* * *}$ & $72.8^{* * *}$ & $71.8^{* * *}$ & $66.9^{* * *}$ & $65.5^{* * *}$ & $13.1^{* * *}$ & $12.4^{* * *}$ & $25.2^{* * *}$ & $24.0^{* * *}$ \\
\hline & $(56.9-65.5)$ & $(55.1-63.7)$ & $(68.5-77.1)$ & $(67.6-76.0)$ & $(62.4-71.4)$ & $(61.1-69.9)$ & $(9.9-16.4)$ & $(9.2-15.6)$ & $(21.0-29.3)$ & $(20.1-27.9)$ \\
\hline \multicolumn{11}{|c|}{ Respondent experienced physical abuse in childhood } \\
\hline \multirow[t]{2}{*}{ No (reference) } & & 47.2 & & 64.0 & & 52.1 & & 7.0 & & 12.2 \\
\hline & & $(44.9-50.9)$ & & $(61.8-66.1)$ & & $(49.9-54.4)$ & & $(5.7-8.3)$ & & $(10.6-13.8)$ \\
\hline \multirow[t]{2}{*}{ Yes } & & 49.9 & & 65.9 & & $56.1^{* *}$ & & 8.2 & & 14.1 \\
\hline & & $(48.4-51.4)$ & & $(64.4-67.3)$ & & $(54.7-57.5)$ & & $(7.4-9.0)$ & & $(13.0-15.2)$ \\
\hline \multicolumn{11}{|c|}{$\begin{array}{l}\text { Respondent experienced psychological abuse in } \\
\text { childhood }\end{array}$} \\
\hline \multirow[t]{2}{*}{ No (reference) } & & 49.3 & & 64.8 & & 55.3 & & 8.0 & & 14.0 \\
\hline & & $(47.7-50.9)$ & & $(63.2-66.4)$ & & $(53.7-56.9)$ & & $(7.1-8.9)$ & & $(12.8-15.2)$ \\
\hline \multirow[t]{2}{*}{ Yes } & & 49.0 & & 66.0 & & 54.5 & & 7.7 & & 13.0 \\
\hline & & $(47.1-50.9)$ & & $(64.2-67.8)$ & & $(52.6-56.3)$ & & $(6.7-8.7)$ & & $(11.7-14.3)$ \\
\hline \multicolumn{11}{|c|}{ Respondent reports father beat her mother } \\
\hline \multirow[t]{2}{*}{ No (reference) } & & 48.4 & & 64.3 & & 54.2 & & 7.0 & & 12.7 \\
\hline & & $(46.6-50.2)$ & & $(62.6-66.0)$ & & $(52.3-56.0)$ & & $(6.1-8.0)$ & & $(11.3-14.1)$ \\
\hline
\end{tabular}


Table 3 Unadjusted and adjusted percentage of women who experienced various symptoms of anxiety, symptoms of psychogenic non-epileptic seizures, and of psychotic disorders, by exposure to intimate partner violence, socioeconomic and demographic characteristics of the respondent, partner's characteristics, and exposure to domestic abuse in the parental household (Continued)

\begin{tabular}{|c|c|c|c|c|c|}
\hline \multirow[t]{2}{*}{ Yes/Don't know } & 49.8 & 66.2 & 55.6 & $8.5^{*}$ & 14.3 \\
\hline & $(48.0-51.2)$ & $(64.6-67.8)$ & $(53.9-57.4)$ & $(7.5-9.5)$ & $(13.1-15.4)$ \\
\hline \multicolumn{6}{|l|}{ Region } \\
\hline \multirow[t]{2}{*}{ La Paz (reference) } & 53.3 & 69.9 & 54.3 & 6.9 & 13.5 \\
\hline & $(50.5-56.1)$ & $(67.6-72.3)$ & $(51.9-56.8)$ & $(5.6-8.1)$ & $(11.4-15.5)$ \\
\hline \multirow[t]{2}{*}{ Chuquisaca } & $43.0^{* *}$ & $60.7^{* * *}$ & 54.5 & 5.2 & $6.7^{* * *}$ \\
\hline & $(37.9-48.2)$ & $(56.4-65.0)$ & $(48.0-61.0)$ & $(3.7-6.8)$ & $(4.7-8.7)$ \\
\hline \multirow[t]{2}{*}{ Cochabamba } & 50.8 & $66.4^{*}$ & $61.0^{* *}$ & $11.3^{* * *}$ & 15.2 \\
\hline & $(48.1-53.6)$ & $(63.3-69.5)$ & $(58.0-64.0)$ & $(9.0-13.7)$ & $(13.3-17.2)$ \\
\hline \multirow[t]{2}{*}{ Oruro } & 49.0 & $63.3^{* *}$ & 50.1 & 6.5 & $6.8^{* * *}$ \\
\hline & $(44.5-53.4)$ & $(59.5-67.2)$ & $(44.6-55.6)$ & $(4.7-8.3)$ & $(4.8-8.8)$ \\
\hline \multirow[t]{2}{*}{ Potosí } & 46.1 & $58.6^{* * *}$ & $45.8^{* * *}$ & 7.7 & 10.7 \\
\hline & $(41.5-50.6)$ & $(54.6-62.5)$ & $(42.2-49.3)$ & $(5.5-9.7)$ & $(8.2-13.2)$ \\
\hline \multirow[t]{2}{*}{ Tarija } & 51.9 & 68.5 & 58.3 & 7.6 & $17.5^{*}$ \\
\hline & $(48.6-55.2)$ & $(64.6-72.4)$ & $(55.2-61.4)$ & $(5.5-9.7)$ & $(14.5-20.5)$ \\
\hline \multirow[t]{2}{*}{ Santa Cruz } & $45.6^{* * *}$ & $64.8^{* *}$ & 55.0 & 6.7 & 16.1 \\
\hline & $(43.0-48.1)$ & $(61.8-67.7)$ & $(52.1-57.9)$ & $(5.4-8.0)$ & $(13.8-18.4)$ \\
\hline \multirow[t]{2}{*}{ Beni } & $46.3^{*}$ & $51.3^{* * *}$ & 58.6 & $11.6^{* *}$ & 12.2 \\
\hline & $(41.0-51.7)$ & $(45.9-56.6)$ & $(54.2-63.0)$ & $(8.3-14.8)$ & $(9.4-14.9)$ \\
\hline \multirow[t]{2}{*}{ Pando } & $44.3^{* *}$ & $61.3^{* *}$ & 59.6 & $12.9^{* * *}$ & $21.1^{* *}$ \\
\hline & $(39.6-49.0)$ & $(56.5-66.1)$ & $(54.4-64.6)$ & $(9.4-16.3)$ & $(16.6-25.7)$ \\
\hline \multicolumn{6}{|l|}{ Residence } \\
\hline \multirow[t]{2}{*}{ Urban (reference) } & 48.5 & 64.9 & 53.1 & 6.9 & 13.7 \\
\hline & $(46.5-50.5)$ & $(62.8-66.9)$ & $(50.7-55.4)$ & $(5.8-8.0)$ & $(12.2-15.2)$ \\
\hline \multirow[t]{2}{*}{ Rural } & 50.1 & 66.0 & 58.0 & 9.0 & 13.4 \\
\hline & $(47.2-53.1)$ & $(63.4-68.6)$ & $(54.7-61.2)$ & $(7.5-10.6)$ & $(11.4-15.4)$ \\
\hline \multicolumn{6}{|l|}{ Education } \\
\hline \multirow[t]{2}{*}{ None (reference) } & 53.1 & 69.4 & 61.1 & 7.5 & 11.3 \\
\hline & $(47.6-58.6)$ & $(64.4-74.4)$ & $(55.4-66.7)$ & $(5.1-9.9)$ & $(7.7-14.9)$ \\
\hline \multirow[t]{2}{*}{ Primary } & 51.0 & 67.5 & 58.9 & 8.2 & 14.7 \\
\hline & $(49.0-53.0)$ & $(65.5-66.7)$ & $(56.7-61.1)$ & $(7.2-9.3)$ & $(13.2-16.3)$ \\
\hline
\end{tabular}


Table 3 Unadjusted and adjusted percentage of women who experienced various symptoms of anxiety, symptoms of psychogenic non-epileptic seizures, and of psychotic disorders, by exposure to intimate partner violence, socioeconomic and demographic characteristics of the respondent, partner's characteristics, and exposure to domestic abuse in the parental household (Continued)

\begin{tabular}{|c|c|c|c|c|c|}
\hline \multirow[t]{2}{*}{ Secondary } & 48.6 & 64.2 & $51.3^{* *}$ & 7.5 & 13.1 \\
\hline & $(46.0-51.2)$ & $(61.7-66.7)$ & $(48.6-54.0)$ & $(6.0-9.0)$ & $(11.2-14.9)$ \\
\hline \multirow[t]{2}{*}{ Higher } & $41.3^{* *}$ & $58.2^{* *}$ & $45.3^{* * *}$ & 6.8 & 10.9 \\
\hline & $(36.3-46.4)$ & $(53.5-62.9)$ & $(40.4-50.3)$ & $(4.3-9.4)$ & $(7.5-14.3)$ \\
\hline \multicolumn{6}{|l|}{ SES } \\
\hline \multirow[t]{2}{*}{ First (reference) } & 50.1 & 66.6 & 57.9 & 9.1 & 11.5 \\
\hline & $(46.3-53.9)$ & $(62.8-70.4)$ & (53.9-61.9) & $(7.0-11.1)$ & $(9.1-14.0)$ \\
\hline \multirow[t]{2}{*}{ Second } & 49.2 & 66.9 & $52.4^{* *}$ & 7.7 & $14.4^{*}$ \\
\hline & $(45.9-52.4)$ & $(63.8-70.1)$ & $(48.2-56.7)$ & $(6.2-9.3)$ & $(12.1-16.8)$ \\
\hline \multirow[t]{2}{*}{ Third } & 51.4 & 67.2 & 55.3 & 7.9 & 15.1 \\
\hline & $(48.7-54.1)$ & $(64.6-69.8)$ & $(52.6-58.0)$ & $(6.3-9.6)$ & $(12.9-17.2)$ \\
\hline \multirow[t]{2}{*}{ Fourth } & 49.1 & 65.3 & 55.2 & 6.5 & 14.3 \\
\hline & $(46.2-52.0)$ & $(62.5-68.0)$ & $(52.1-58.4)$ & $(4.9-8.2)$ & $(12.3-16.3)$ \\
\hline \multirow[t]{2}{*}{ Fifth } & 45.8 & 60.8 & 54.0 & 7.8 & 12.0 \\
\hline & $(42.2-49.5)$ & $(57.6-64.0)$ & $(50.1-57.9)$ & $(5.7-9.9)$ & $(9.5-14.5)$ \\
\hline \multicolumn{6}{|l|}{ Work status } \\
\hline \multirow[t]{2}{*}{ Not working (reference) } & 47.8 & 65.5 & 55.4 & 7.0 & 12.4 \\
\hline & $(45.7-49.9)$ & $(63.4-67.6)$ & $(53.3-57.4)$ & $(6.0-8.1)$ & $(11.0-13.8)$ \\
\hline \multirow[t]{2}{*}{ Working } & 49.8 & 65.2 & 54.7 & 8.3 & $14.2^{*}$ \\
\hline & $(48.3-51.4)$ & $(63.7-66.7)$ & $(53.3-56.2)$ & $(7.4-9.2)$ & $(13.1-15.3)$ \\
\hline \multicolumn{6}{|l|}{ Age } \\
\hline \multirow[t]{2}{*}{ 15-24 (reference) } & 49.4 & 66.2 & 47.3 & 5.8 & 13.2 \\
\hline & (44.9-53.8) & $(61.8-70.6)$ & $(43.1-51.5)$ & $(6.8-8.5)$ & $(10.3-16.0)$ \\
\hline \multirow[t]{2}{*}{$25-34$} & 50.1 & 65.5 & $54.8^{* *}$ & 7.2 & 14.2 \\
\hline & $(47.7-52.4)$ & $(63.3-67.7)$ & $(52.5-57.2)$ & $(6.1-8.3)$ & $(12.6-15.7)$ \\
\hline \multirow[t]{2}{*}{$35-44$} & 47.1 & 64.5 & $57.4^{* *}$ & 8.6 & 13.0 \\
\hline & $(44.4-49.8)$ & $(62.0-67.1)$ & $(54.7-60.1)$ & $(7.1-10.1)$ & $(11.1-14.9)$ \\
\hline \multirow[t]{2}{*}{$45-49$} & 51.5 & 65.4 & $59.8^{* *}$ & $11.3^{*}$ & 13.9 \\
\hline & $(46.8-56.2)$ & $(61.2-69.7)$ & $(55.1-64.4)$ & $(8.2-14.5)$ & $(10.4-17.3)$ \\
\hline
\end{tabular}


Table 3 Unadjusted and adjusted percentage of women who experienced various symptoms of anxiety, symptoms of psychogenic non-epileptic seizures, and of psychotic disorders, by exposure to intimate partner violence, socioeconomic and demographic characteristics of the respondent, partner's characteristics, and exposure to domestic abuse in the parental household (Continued)

\begin{tabular}{|c|c|c|c|c|c|}
\hline \multicolumn{6}{|l|}{ Marital status } \\
\hline \multirow[t]{2}{*}{ Married (reference) } & 49.6 & 65.3 & 54.6 & 7.7 & 13.3 \\
\hline & $(48.0-51.3)$ & $(63.5-67.1)$ & $(43.0-56.2)$ & $(6.8-8.5)$ & $(12.2-14.5)$ \\
\hline \multirow[t]{2}{*}{ Cohabiting } & 48.4 & 65.3 & 54.7 & 8.1 & 13.9 \\
\hline & $(46.3-50.4)$ & $(63.1-67.5)$ & $(54.5-57.5)$ & $(6.9-9.4)$ & $(12.4-15.4)$ \\
\hline \multicolumn{6}{|l|}{ Partner's age } \\
\hline \multirow[t]{2}{*}{ 14-24 (reference) } & 50.5 & 67.2 & 54.8 & 9.7 & 18.8 \\
\hline & $(44.9-56.2)$ & $(62.0-72.4)$ & $(49.6-60.0)$ & $(6.2-13.3)$ & $(13.8-23.9)$ \\
\hline \multirow[t]{2}{*}{$25-34$} & 49.5 & 65.3 & 53.0 & 8.2 & $13.7^{*}$ \\
\hline & $(46.8-52.2)$ & $(62.9-67.8)$ & $(50.2-55.8)$ & $(6.5-9.9)$ & $(11.8-15.6)$ \\
\hline \multirow[t]{2}{*}{$35-44$} & 47.4 & 64.2 & 55.5 & 8.0 & $12.5^{*}$ \\
\hline & $(47.1-52.0)$ & $(62.0-66.4)$ & $(53.3-57.7)$ & $(6.9-9.1)$ & $(10.9-14.0)$ \\
\hline \multirow[t]{2}{*}{$45+$} & 47.4 & 66.1 & 57.3 & 6.8 & 12.7 \\
\hline & $(43.4-51.5)$ & $(62.2-69.9)$ & $(53.4-61.2)$ & $(5.1-8.5)$ & $(10.1-15.3)$ \\
\hline \multicolumn{6}{|c|}{ Partner's age, relative to respondent } \\
\hline \multirow[t]{2}{*}{ Same age or younger (ref.) } & 50.3 & 65.9 & 57.2 & 6.8 & 14.3 \\
\hline & $(47.7-52.9)$ & $(63.4-68.4)$ & $(54.8-59.6)$ & $(5.6-8.0)$ & $(12.6-16.0)$ \\
\hline \multirow[t]{2}{*}{ 1-5 years older } & 49.5 & 65.9 & $53.7^{*}$ & 7.7 & 12.8 \\
\hline & $(47.6-51.4)$ & $(64.0-67.7)$ & $(51.9-55.4)$ & $(6.7-8.7)$ & $(11.6-14.0)$ \\
\hline \multirow[t]{2}{*}{$6+$ years older } & 47.4 & 63.7 & 54.6 & $9.4^{*}$ & 14.0 \\
\hline & $(44.7-50.1)$ & $(61.2-66.3)$ & $(51.7-57.5)$ & $(7.7-11.0)$ & $(12.1-16.0)$ \\
\hline \multicolumn{6}{|l|}{ Partner's education } \\
\hline \multirow[t]{2}{*}{ None (reference) } & 47.9 & 63.7 & 53.2 & 7.0 & 16.9 \\
\hline & $(38.8-57.0)$ & $(54.7-72.7)$ & $(43.9-62.4)$ & $(1.8-12.2)$ & $(8.3-25.6)$ \\
\hline \multirow[t]{2}{*}{ Primary } & 50.1 & 65.7 & 55.4 & 8.7 & 14.7 \\
\hline & $(47.6-52.7)$ & $(63.5-67.8)$ & $(53.0-57.8)$ & $(7.4-10.0)$ & $(13.0-16.4)$ \\
\hline \multirow[t]{2}{*}{ Secondary } & 50.7 & 66.5 & 55.1 & 7.0 & 12.7 \\
\hline & $(48.5-52.8)$ & $(64.3-68.8)$ & $(53.0-57.1)$ & $(6.0-8.1)$ & $(11.1-14.3)$ \\
\hline \multirow[t]{2}{*}{ Higher } & 43.8 & 62.3 & 53.8 & 7.0 & 12.3 \\
\hline & $(39.5-48.1)$ & $(58.6-66.1)$ & $(49.7-58.0)$ & $(5.0-9.1)$ & $(9.4-15.1)$ \\
\hline
\end{tabular}


Table 3 Unadjusted and adjusted percentage of women who experienced various symptoms of anxiety, symptoms of psychogenic non-epileptic seizures, and of psychotic disorders, by exposure to intimate partner violence, socioeconomic and demographic characteristics of the respondent, partner's characteristics, and exposure to domestic abuse in the parental household (Continued)

\begin{tabular}{|c|c|c|c|c|c|}
\hline \multicolumn{6}{|c|}{ Partner's education compared to respondent } \\
\hline \multirow[t]{2}{*}{ Wife is more educated (reference) } & 47.9 & 63.6 & 55.2 & 7.5 & 12.2 \\
\hline & $(44.9-50.9)$ & $(60.9-66.4)$ & $(52.4-58.0)$ & $(5.9-9.0)$ & $(10.3-14.1)$ \\
\hline \multirow[t]{2}{*}{ Same level of education } & 46.9 & 63.5 & 54.6 & 6.7 & 13.8 \\
\hline & $(44.4-49.4)$ & $(61.0-65.9)$ & $(52.1-57.0)$ & $(5.4-8.0)$ & $(12.0-15.5)$ \\
\hline \multirow[t]{2}{*}{ Husband is more educated } & 50.6 & 66.8 & 55.0 & 8.4 & 14.1 \\
\hline & $(48.7-52.6)$ & $(65.0-68.6)$ & $(53.1-56.9)$ & $(7.3-9.5)$ & $(12.7-15.4)$ \\
\hline
\end{tabular}

${ }^{*} p<0.05 * * p<0.01 * * * p<0.001$.

$\psi$ Respondents who experienced sexual abuse may also have experienced psychological and/or physical abuse. 


\section{Conclusions}

Our findings show that there is an urgent need for studies on the prevalence and health consequences of psychological intimate partner violence in other developing countries. Because evidence is building that IPV-related mental health problems are particularly acute among Latina women [62-64], more research on Latin American countries is particularly warranted. The finding that women who only experienced psychological abuse by an intimate partner report have nearly identical symptoms of mental health problems as women who were physically abused has important programmatic implications. Specifically, it highlights the importance of ensuring that victims of intimate partner violence have adequate access to mental health services. Research on Hispanic women in the U.S. has also emphasized the need for culturally appropriate outreach programs to increase awareness of the negative effects of intimate partner violence on women's mental health and to increase women's awareness about how to access mental health services [63]. The finding that physical abuse and psychological abuse are often experienced concurrently further suggests that health providers who treat victims of physical intimate partner violence should also screen these patients for symptoms of potential mental health problems and - if needed - refer them to appropriate mental health services.

\section{Competing interests}

The authors declare that they have no competing interests.

\section{Authors' contributions}

DM and SP conducted the data analysis and prepared the first draft of the manuscript. PH contributed to the analysis plan and made critical revisions to the methodology. All authors read and approved the final manuscript.

\section{Acknowledgments}

This study uses data from the 2008 Bolivia Demographic and Health Survey, which was funded by the United States Agency for International Development (USAID). Preliminary results of the study were presented at the Annual Meeting of the Population Association of America, held in San Francisco, May 2-5, 2012.

Received: 27 July 2012 Accepted: 20 June 2013

Published: 26 June 2013

\section{References}

1. Gender based violence in Latin America. http:/www.smartglobalhealth.org/ blog/entry/gender-based-violence-in-Latin-America/.

2. Díaz-Olavarrieta C, Wilson KS, García SG, Revollo R, Richmond K, Paz F, Chavez $L P$ : The co-occurrence of intimate partner violence and syphilis among pregnant women in Bolivia. J Womens Health 2009, 18(12):2077-2086.

3. Ellsberg M, Jansen HAFM, Heise L, Watts CH, Garcia-Moreno C: Intimate partner violence and women's physical and mental health in the WHO multi-country study on women's health and domestic violence: an observational study. Lancet 2008, 371(9619):1165-1172.

4. García-Moreno C, Jansen HAFM, Ellsberg M, Heise L, Watts CH: Prevalence of intimate partner violence: findings from the WHO multi-country study on women's health and domestic violence. Lancet 2006, 368(9543):1260-1269.

5. Pallitto CC, O'Campo P: The relationship between intimate partner violence and unintended pregnancy: analysis of a national sample from Colombia. Int Fam Plan Perspect 2004, 30(4):165-173.
6. Heise L, Ellsberg M, Gottmoeller M: A global overview of gender-based violence. International Journal of Gynecology and Obstetrics 2002, 78(Suppl. 1):S5-S14.

7. Hindin MJ, Kishor S, Ansara DL: Intimate partner violence among couples in 10 DHS countries: Predictors and health outcomes. Calverton, MD: Macro International Incorporated; 2008.

8. Parish W, Laumann E, Pan S, Luo Y: Intimate partner violence in China: national prevalence, risk factors and associated health problems. Int Fam Plan Perspect 2004, 30(4):174-191.

9. Population Reference Bureau, Interagency Gender Working Group: Gender-based violence: impediment to reproductive health. Washington, D.C: Population Reference Bureau; 2010.

10. Downs W, Capshew T, Rindels B: Relationships between adult women's alcohol problems and their childhood experiences of parental violence and psychological aggression. Violence Against Women 2004, 8:785-815.

11. Kaplan LM, Hill TD, Mann-Deibert GR: Does alcohol consumption exacerbate the mental health consequence of domestic violence? Violence Against Women 2012, 18(3):289-308.

12. Kaukinen C, DeMaris A: Age at first sexual assault and current substance use and depression. J Interpers Violence 2005, 20:1244-1270.

13. Springer K, Sheridan J, Kuo D, Carnes M: Long-term physical and mental health consequences of childhood sexual abuse: results from a large populationbased sample of men and women. Child Abuse Negl 2007, 31:289-308.

14. Tolman R, Rosen D: Domestic violence in the lives of women receiving welfare: mental health, substance dependence, and well-being. Violence Against Women 2001, 7:141-158.

15. Zlotnik C, Johnson D, Kohn R: Intimate partner violence and long-term psychosocial functioning in a national sample of American women. J Interpers Violence 2006, 21:262-275.

16. Ishida K, Stupp P, Melian M, Serbanescu F, Goodwin M: Exploring the associations between intimate partner violence and women's mental health: evidence from a population-based study in Paraguay. Soc Sci Med 2010, 71(9):1653-1661.

17. Avanci J, Assis S, Oliveira R: A cross-sectional analysis of women's mental health problems: examining the association with different types of violence among a sample of Brazilian mothers. BMC Womens Health 2013, 13:20.

18. Fischbach RL, Herbert B: Domestic violence and mental health: correlates and conundrums within and across cultures. Soc Sci Med 1997, 45(8):1161-1176

19. United Nations: Declaration on the elimination of violence against women. ARES/ 48/104 adopted on December 20, 1993. New York: UN General Assembly; 1993.

20. García-Moreno C, Jansen HAFM, Ellsberg M, Heise L, Watts C: WHO multicountry study on women's health and domestic violence against women: initial results on prevalence, health outcomes and women's responses. Geneva: World Health Organization; 2005.

21. Bott S, Morrison A, Ellsberg M: Preventing and responding to gender-based violence in middle and low-income countries: a global review and analysis. Washington, D.C.: World Bank; 2005

22. Díaz-Olavarrieta C, García SG, Feldman BS, Polis AM, Revollo R, Tinajeros F, Grossman D: Maternal syphilis and intimate partner violence in Bolivia: a gender-based analysis of implications for partner notification and universal screening. Sex Transm Dis 2007, 34(7):S42.

23. Stieglitz J, Kaplan H, Gurven M, Winking J, Tayo BV: Spousal violence and paternal disinvestment among Tsimane'forager-horticulturalists. Am J Hum Biol 2011, 24(4):445-457.

24. Abramsky T, Watts C, Garcia-Moreno C, Devries K, Kiss L, Ellsberg M, Jansen $H$, Heise $L$ : What factors are associated with recent intimate partner violence? findings from the WHO multi-country study on women's health and domestic violence. BMC Publ Health 2011, 11(1):109.

25. Brownridge DA, Halli SS: "Living in sin" and sinful living Toward filling a gap in the explanation of violence against women. Aggression and Violent Behavior 2000, 5(6):565-583.

26. Dalal K, Lindqvist K: A National study of the prevalence and correlates of domestic violence among women in India. Asia Pac J Public Health 2012, 24(2):265-277.

27. Flake DF, Forste R: Fighting families: family characteristics associated with domestic violence in five Latin American countries. Journal of Family Violence 2006, 21(1):19-29.

28. Fehringer JA, Hindin MJ: Like parent, like child: intergenerational transmission of partner violence in Cebu, the Philippines. J Adolesc Health 2009, 44(4):363-371. 
29. Jewkes R, Levin J, Penn-Kekana L: Risk factors for domestic violence: findings from a South African cross-sectional study. Soc Sci Med 2002, 55(9):1603-1617.

30. Kishor S, Johnson K: Profiling domestic violence: a multi-country study. Calverton, MD: MEASURE DHS, ORC Macro; 2004.

31. Martin SL, Moracco KE, Garro J, Tsui AO, Kupper LL, Chase JL, Campbell JC: Domestic violence across generations: findings from northern India. Int J Epidemiol 2002, 31(3):560-572.

32. Koenig MA, Lutalo T, Zhao F, Nalugoda F, Wabwire-Mangen F, Kiwanuka N, Wagman J, Serwadda D, Wawer M, Gray R: Domestic violence in rural Uganda: evidence from a community-based study. Bull World Health Organ 2003, 81(1):53-60.

33. Coa R, Ochoa L: Bolivia Encuesta Nacional de Demografía y Salud 2008 (ENDSA 2008). La Paz, Bolivia: Ministerio de Salud y Deportes, Instituto Nacional de Estadīstica; 2009.

34. World Health Organization: A user's guide to the self reporting questionnaire (SRQ). Geneva: Division of Mental Health, World Health Organization; 1994

35. Sattar A, Salih M, Jafri W: Burden of common mental disorders in patients with Functional Dyspepsia. J Pak Med Assoc 2010, 60(12):995-997.

36. Mocellin J, AA Coêlho J: Psychological responses to drought in Northeastern Brazil. Interamerican Journal of Psychology 2004, 38(1):95-103.

37. Nakimuli-Mpungu E, Mutamba B, Othengo M, Musisi S: Psychological distress and adherence to highly active anti-retroviral therapy (HAART) in Uganda: a pilot study. Afr Health Sci 2009, 9(Special Issue 1):S2-S7.

38. de Jong K, van der Kam S, Ford N, Lokuge K, Fromm S, van Galen R, Reilley B, Kleber R: Conflict in the Indian Kashmir Valley II: psychological impact. Confl Heal 2008, 2(11). doi:10.1186/1752-1505-2-11.

39. Wutich A, Ragsdale K: Water insecurity and emotional distress: coping with supply, access, and seasonal variability of water in a Bolivian squatter settlement. Soc Sci Med 2008, 67:2116-2125.

40. Irmansyah I, Dharmono S, Maramis A, Minas H: Determinants of psychological morbidity in survivors of the earthquake and tsunami in Aceh and Nias. Int J Ment Heal Syst 2010, 4:8.

41. Bordin I, Duarte C, Peres C, Nascimento R, Curto B, Paula C: Severe physical punishment: risk of mental health problems for poor urban children in Brazil. Bull World Health Organ 2008, 87:336-344

42. Tuan T, Harpham T, Huong NT: Validity and reliability of the self-reporting questionnaire 20 items in Vietnam. Hong Kong Psychiatry 2004, 14(3):15-18.

43. Hanlon C, Medhin G, Alem A, Araya M, Abdulahi A, Hughes M, Tesfaye M, Wondimagegn $D$, Patel $V$, Prince M: Detecting perinatal common mental disorders in Ethiopia: validation of the self-reporting questionnaire and Edinburgh postnatal depression scale. J Affect Disord 2008, 108:251-262.

44. de Avedo-Marques J M: Zuardi AW: COOP/WONCA Charts as a screen for mental disorders in primary care. Annuals of Family Medicine 2011, 9:359-365.

45. Harpham T, Reichenheim M, Oser R, Thomas E, Hamid N, Jaswal S, Ludermir A, Aidoo M: Measuring mental health in a cost-effective manner. Health Policy Plan 2003, 18(3):344-349.

46. Kinyanda E, Hielmeland H, Musisi S: Psychological factors in deliberate self-harm as seen in an urban African population in Uganda: a case-control study. Suicide Life Threat Behav 2005, 34(4):468-477.

47. Kinyanda E, Waswa L, Baisley K, Maher D: Prevalence of severe mental distress and its correlates in a population-based study in rural south-west Uganda. BMC Psychiatry 2011, 11:07.

48. Abbo C, Ekblad S, Waako P, Elialilia O, Musisi S: The prevalence and severity of mental illness handled by traditional healers in two districts in Uganda. Afr Health Sci 2009, 9(Special Issue 1):S16-S22.

49. Ramli H, Ohn K, Krishnaswamy S, Kasmini K, Hassan S: A prevalence survey of psychiatric morbidity in a rural Malaysian village: a preliminary report. Singapore Med J 1987, 28(6):530-533.

50. Vos T, Astbury J, Piers LS, Magnus A, Heenan M, Stanley L, Walker L, Webster $\mathrm{K}$ : Measuring the impact of intimate partner violence on the health of women in Victoria, Australia. Bull World Health Organ 2006, 84(9):739-744.

51. Kishor S, Bradley SEK: Women's and men's experience of spousal violence in two African countries: does gender matter? (DHS Analytical Studies, No. 27). Calverton, MD: ICF International Inc.; 2012.

52. Rutstein SO, Johnson K: The DHS wealth index (DHS Comparative Reports, No. 6). ORC Macro: Calverton, MD; 2004

53. Greene WH: Econometric Analysis. New York: Prentice-Hall; 2002.

54. Williams R: Using the margins command to estimate and interpret adjusted predictions and marginal effects. Stata J 2012, 12(2):308-331.
55. Dillon G, Hussain R, Loxton D, Rahman S: Mental and physical health and intimate partner violence against women: a review of the literature. International journal of family medicine 2013, 2013:313909.

56. Beydoun HA, Beydoun MA, Kaufman JS, Lo B, Zonderman AB: Intimate partner violence against adult women and its association with major depressive disorder, depressive symptoms and postpartum depression: a systematic review and meta-analysis. Soc Sci Med 2012, 75(6):959-975.

57. Gomez-Beloz A, Williams MA, Sanchez SE, Lam N: Intimate partner violence and risk for depression among postpartum women in Lima, Peru. Violence Vict 2009, 24(3):380-398.

58. Hegarty K, Gunn J, Chondros P, Small R: Association between depression and abuse by partners of women attending general practice: descriptive, cross sectional survey. BMJ 2004, 328(7440):621-624

59. Houry D, Kemball R, Rhodes KV, Kaslow NJ: Intimate partner violence and mental health symptoms in African American female ED patients. Am J Emerg Med 2006, 24(4):444-450.

60. Ludermir AB, Lewis G, Valongueiro SA, de Araujo TV, Araya R: Violence against women by their intimate partner during pregnancy and postnatal depression: a prospective cohort study. Lancet 2010 376(9744):903-910

61. Rodriguez MA, Heilemann MV, Fielder E, Ang A, Nevarez F, Mangione CM: Intimate partner violence, depression, and PTSD among pregnant Latina women. Ann Fam Med 2008, 6(1):44-52.

62. Bonomi AE, Anderson ML, Cannon EA, Slesnick N, Rodriguez MA: Intimate partner violence in Latina and non-Latina women. Am J Prev Med 2009, 36(1):43-48.

63. Lipsky S, Caetano R: Impact of intimate partner violence on unmet need for mental health care: results from the NSDUH. Psychiatr Serv 2007, 58(6):822-829.

64. Lipsky S, Caetano R, Roy-Byrne P: Racial and ethnic disparities in policereported intimate partner violence and risk of hospitalization among women. Women's health issues: official publication of the Jacobs Institute of Women's Health 2009, 19(2):109-118.

65. Pico-Alfonso MA, Garcia-Linares MI, Celda-Navarro N, Blasco-Ros C, Echeburua E, Martinez M: The impact of physical, psychological, and sexual intimate male partner violence on women's mental health: depressive symptoms, posttraumatic stress disorder, state anxiety, and suicide. J Womens Health (Larchmt) 2006, 15(5):599-611.

66. Coker AL, Smith PH, Thompson MP, McKeown RE, Bethea L, Davis KE: Social support protects against the negative effects of partner violence on mental health. J Womens Health Gend Based Med 2002, 11(5):465-476.

67. Vaeth PA, Ramisetty-Mikler S, Caetano R: Depression among couples in the United States in the context of intimate partner violence. $J$ Interpers Violence 2010, 25(5):771-790.

doi:10.1186/1472-6874-13-28

Cite this article as: Meekers et al.: Intimate partner violence and mental health in Bolivia. BMC Women's Health 2013 13:28.

\section{Submit your next manuscript to BioMed Central and take full advantage of:}

- Convenient online submission

- Thorough peer review

- No space constraints or color figure charges

- Immediate publication on acceptance

- Inclusion in PubMed, CAS, Scopus and Google Scholar

- Research which is freely available for redistribution 